\title{
A systematic review of studies measuring health-related quality of life of general injury populations
}

\author{
Suzanne Polinder ${ }^{1 *}$, Juanita A Haagsma ${ }^{1}$, Eefje Belt ${ }^{1}$, Ronan A Lyons², Vicki Erasmus ${ }^{1}$, Johan Lund ${ }^{3}$,
} Ed F van Beeck ${ }^{1}$

\begin{abstract}
Background: It is important to obtain greater insight into health-related quality of life (HRQL) of injury patients in order to document people's pathways to recovery and to quantify the impact of injury on population health over time. We performed a systematic review of studies measuring HRQL in general injury populations with a generic health state measure to summarize existing knowledge.

Methods: Injury studies (1995-2009) were identified with main inclusion criteria being the use of a generic health status measure and not being restricted to one specific type of injury. Articles were collated by study design, HRQL instrument used, timing of assessment(s), predictive variables and ability to detect change over time.

Results: Forty one studies met inclusion criteria, using 24 different generic HRQL and functional status measures (most used were SF-36, FIM, GOS, EQ-5D). The majority of the studies used a longitudinal design, but with different lengths and timings of follow-up (mostly 6, 12, and 24 months). Different generic health measures were able to discriminate between the health status of subgroups and picked up changes in health status between discharge and 12 month follow-up. Most studies reported high prevalences of health problems within the first year after injury. The twelve studies that reported HRQL utility scores showed considerable but incomplete recovery in the first year after discharge.

Conclusion: This systematic review demonstrates large variation in use of HRQL instruments, study populations, and assessment time points used in studies measuring HRQL of general injury populations. This variability impedes comparison of HRQL summary scores between studies and prevented formal meta-analyses aiming to quantify and improve precision of the impact of injury on population health over time.
\end{abstract}

\section{Background}

Worldwide, injuries are recognized as a major concern in public health, being the predominant cause of deaths in adults aged 1- 45 years, and an important cause of disabilities [1,2]. The number of survivors of severe injuries has rapidly grown due to substantial improvements in trauma care. This has resulted in a shift of focus from mortality towards disability of injury patients. Disability (i.e. reduced levels of functioning resulting from diseases or injuries [3]) is increasingly seen as an

\footnotetext{
* Correspondence: s.polinder@erasmusmc.nl

'Erasmus Medical Centre, Department of Public Health, Rotterdam, the Netherlands

Full list of author information is available at the end of the article
}

important component of a population's health and for the field of injury prevention and trauma care [4].

Disability is a complex construct and can be measured using functional instruments or generic or disease specific HRQL measures, where disability represents the gap between measured and perfect HRQL. To enable straightforward comparisons with other disease groups and with general population norms, it is necessary to measure the consequences of injuries using generic health status measures (for instance the SF-36 or the EQ-5D). Some HRQL instruments generate a summary score (utility) that can contribute to a composite health outcome measure [1]. It has become common practice to quantify the impact of diseases and injuries on
Ciomed Central

() 2010 Polinder et al; licensee BioMed Central Ltd. This is an Open Access article distributed under the terms of the Creative Commons Attribution License (http://creativecommons.org/licenses/by/2.0), which permits unrestricted use, distribution, and reproduction in any medium, provided the original work is properly cited. 
population health with the help of composite health outcome measures, such as quality-adjusted life years (QALYs) and disability-adjusted life years (DALYs) $[4,5]$. Sound epidemiological data on the incidence, severity and duration of the functional consequences of injuries are needed to make valid estimates of the years lived with disability due to injuries in the population. Data on all dimensions of functioning relevant to injuries are needed to describe the pattern of recovery or residual disability of injury patients over time. With the help of these data, the impact of injury on population health over time can be quantified. Measuring the impact of injury is particularly challenging due to the large variation in injury types and severity. The European Consumer Safety Association has published guidelines for the conduction of follow-up studies measuring injury-related disability based on a narrative literature search of papers from1995-2005 [1]. They concluded that in the injury field there is lack of consensus on preferred HRQL instruments and study designs [1]. However, this review only included 14 studies that measured HRQL in general injury populations. Derrett et al conducted a more recent systematic literature search of injury specific and generic studies measuring outcome after injury but restricted this to studies using the EQ-5D outcome measure. They called for further comprehensive population-level research exploring outcomes after injury, and particularly for studies focusing on 'all injury' [6]. It is clear that there is a need to obtain greater insight into patterns of HRQL in comprehensive injury populations in order to document people's pathways to recovery and to quantify the impact of injury on population health over time $[1,6,7]$. Given the appearance of additional studies after 2005 , and the variety of generic measures in this field of research, the current systematic review was conducted to describe the up to date state of knowledge in this field and hopefully contribute to further consensus development on preferred methodologies within the injury research field.

This review focused on the measurement of HRQL with a generic instrument among general injury populations. The following key questions were addressed: a) which generic instruments were used?, b) how were these instruments administered?, c) at which time points was HRQL assessed?, and d) did the instrument measure changes over time and predictors for HRQL? Furthermore, in anticipation of substantial heterogeneity preventing formal meta-analysis we aimed to produce a narrative summary of study outcomes to improve insight into general recovery patterns and residual disability.

\section{Methods}

\section{Data sources and search strategy}

We conducted a literature search aiming to identify empirical studies on injury-related disability. Searches of eligible studies were conducted in PubMed (Medline), Web of Science, Embase, and PsychInfo. All peerreviewed articles published in the period January 1995 to 2009 were included in the searches. An electronic search strategy was developed in collaboration with a librarian with extensive experience in systematic reviews. Search terms used were: 'wounds and injuries', 'health status indicators', 'disability evaluation', 'functional outcome', 'health status measure', and 'cohort studies' (details in Additional file 1). Keywords were matched to database specific indexing terms. In addition to database searches, reference lists of review studies and articles included in the review were screened for titles that included key terms. More detailed information on the review can be found in the report compiled for the INTEGRIS (Integration of European Injury Statistics) project [8].

\section{Selection criteria}

The inclusion criteria were studies using HRQL instruments in injury patients irrespective of the underlying injury, published in English or German in a peerreviewed journal in the period 1995-2009. We focused on 'all injury' studies and therefore excluded injuryspecific studies (for instance limited to brain injuries or hip fractures). Studies concerning people other than the injury victim were excluded, e.g. studies of impact of witnessing trauma. We included studies that reflected the definition of injuries used by the World Health Organization (WHO) as 'relatively sudden discernible effects due to body tissue damage from energy exchanges or ingestion of toxic substances but not due to medical adverse events, and obtained from health care settings' [9]. We included only longitudinal studies in line with the EuroSafe guidance [1].

\section{Data extraction}

Relevant papers were selected by screening the titles (first step), abstracts (second step) and entire articles (third step), retrieved through the database searches. During each step the title, abstract or entire article was screened to ensure that it met the selection criteria listed above. This screening was conducted independently by two researchers (SP and EB). Two experts in this field (RL and JL) checked a sample of the abstracts $(n=50)$ on the inclusion criteria, to quality assure the process. Full articles were critically appraised by two reviewers (EB and SP), using data extraction forms developed for this study in a Microsoft Access-database.

Data were tabulated from studies that used a HRQL instrument and that reported a utility score or summary score, to give greater insight into the recovery patterns and changes over time of the different instruments. Utility scores are based on preferences or values related to 
health states and are derived from approaches used in decision theory and economics. Utility scores represent the total HRQL status of a person in a number on a 0 (or $<0$ )-1 scale (where 0 indicates death or maximum amount of disability, and 1 being optimal health status). The EQ-5D and the SF-6D are examples of HRQL assessment instruments that produce such utility scores. Some instruments report a disability score (in which 1 represents the maximum amount of disability) for instance the WHODAS II [10]. Although there are some differences between the concepts, utility and disability scores will be referred to as summary scores in the remainder of this paper.

\section{Results}

\section{Literature search}

The database search identified 6291 titles of potentially relevant articles. In the first round (scanning the titles) 6031 articles were excluded. The main reasons for exclusion were studies which did not concern injury or were restricted to specific injuries. Of the remaining 260 articles, 165 were excluded after scanning the abstracts, mainly because the paper did not include self-reported HRQL measures. This resulted in scanning 95 full texts of which 54 did not meet our inclusion criteria, leading to inclusion of 41 articles. In this last round the main reason for excluding a full-text article was not using a generic health status measure or not describing the general injured population in sufficient detail (Additional file 2).

\section{Study characteristics}

Of the 41 studies included in our systematic review, most were conducted in the US $(\mathrm{n}=12$, [11-22]), followed by the UK ( $\mathrm{n}=7,[23-29])$, Australia $(\mathrm{n}=5$, [30-33]), and the Netherlands ( $\mathrm{n}=5$, [34-38]) (Table 1 and 2). Sample sizes of the studies varied widely, between 35 and 13,649 participants, with most studies having sample sizes between 100 and 300 participants ( $\mathrm{n}=17$, Table 1 and 2 ). Nine studies measured HRQL after injury for children and adolescents (shaded articles in table 1 and 2). All studies included non selected cases from 'all injury' populations and included injuries of different levels of severity.

Studies of disability restricted to the most severely injured patients are increasingly conducted. These studies used different severity scales and cutoff points for decision criteria for 'major trauma patients'. There were 10 studies that clearly described inclusion criteria using the most widely used inclusion definition of major trauma patients, namely an Injury Severity Score (ISS) > 15. Threshold scores from the Abbreviated Injury Scale (AIS), Glasgow Coma Score (GCS) or admission to a 'trauma center' for longer than 24 hours were also used as inclusion criteria.
Particularly for studies of low to moderate severity injury populations, e.g. emergency department (ED) attendees, there were difficulties in acquiring acceptable response and retention rates. Higher rates were more often reported in studies where outcome measures were administered by clinicians.

\section{Measurement of health related quality of life Study design}

Twenty-four different instruments were used to assess HRQL or functional status. Of the available generic instruments, the SF-36 $(\mathrm{n}=15)$, (Wee)FIM $(\mathrm{n}=10)$, GOS $(n=7)$ and the EQ-5D $(n=5)$ have most often been applied among injury patients (see Figure 1). Half of the studies used more than one instrument to measure HRQL (two instruments: $\mathrm{n}=10$; and more than two instruments: $\mathrm{n}=10$ ). None of these studies used an injury specific measure besides a generic measure. In the nine studies among children, only three used a children's instrument $[21,39,40]$. All three studies also included an 'all-ages' instrument.

Twenty-six studies used a longitudinal design with multiple assessments over time. HRQL was assessed most frequently at discharge, six months, one year, and two years following injury (Figure 2). There were five papers that assessed pre-injury health status, i.e. after the injury patients experienced the shock of sustaining an injury $[14,15,33,41]$. Variation was also apparent in the mode of administration with a mixture of self-completed questionnaires $(\mathrm{n}=14)$, face to face interviews $(\mathrm{n}=13)$, and telephone interviews $(n=14)$ of which 4 were telephone proxy interviews with parents, being used.

\section{Predictors for HRQL}

High prevalence's of health problems within the first year after injury were a common finding of the studies. Studies often included a large variety of associated variables which affected disability scores. Predictive variables frequently reported included injury severity, type of injury, gender, mental health status and comorbidity. The generic instruments showed similar differences between subgroups. The SF-36 and the EQ-5D were reported to be able to discriminate between the health status of injured patients and non-injured persons and between patients with different types of injuries (e.g. $[13,33,42,43])$. Among the majority of studies hospitalization, injury type and/or mechanism, and injury severity were predictive for long-term disability.

\section{Changes over time}

All HRQL instruments demonstrated improvements in health over time within the first 3 to 6 months after the injury. Most studies reported improvement in HRQL between discharge and one year after injury, and studies of 'severe' injuries also found improvements one to two years following the injury (Table 1). 
Table 1 Study characteristics and methodological aspects of follow-up studies measuring health-related quality of life of injury patients (in order of nr of HRQL instruments used - bold author names are studies of children)

\begin{tabular}{|c|c|c|c|c|c|}
\hline $\begin{array}{l}\text { Author, year, } \\
\text { country }\end{array}$ & $\begin{array}{l}\text { Study } \\
\text { population }\end{array}$ & $\begin{array}{l}\text { HRQL } \\
\text { instrument } \\
\text { (mode of } \\
\text { administration) }\end{array}$ & $\begin{array}{l}\text { Follow up } \\
\text { (time } \\
\text { points and } \\
\text { response } \\
\text { rates) }\end{array}$ & Changes over time & Predictors for HRQL \\
\hline $\begin{array}{l}\text { Meerding, } \\
\text { 2004, } \\
\text { Netherlands } \\
{[35]}\end{array}$ & $\begin{array}{l}\text { ED and/or } \\
\text { admitted } \\
\text { Age } 15+ \\
(\mathrm{n}=4639)\end{array}$ & $\begin{array}{l}\text { EQ-5D } \\
\text { (Self-completed) }\end{array}$ & $\begin{array}{l}2 \text { months } \\
(39 \%) \\
5 \text { months } \\
(24 \%) \\
9 \text { months } \\
(12 \%)\end{array}$ & $\begin{array}{l}\text { Improvements between } 2 \text { and } \\
5 \text { months, no further improvement } \\
\text { between } 5 \text { and } 9 \text { months }\end{array}$ & HRQL associated with body region injured \\
\hline $\begin{array}{l}\text { Polinder, } \\
\text { 2005, } \\
\text { Netherlands } \\
{[36]}\end{array}$ & $\begin{array}{l}\text { ED and/or } \\
\text { admitted } \\
\text { Age 5-14 } \\
(\mathrm{n}=1221)\end{array}$ & $\begin{array}{l}\text { EQ-5D } \\
\text { (Self-completed, } \\
\text { age }<13 \text { proxy) }\end{array}$ & $\begin{array}{l}2.5 \text { month } \\
(43 \%) \\
5 \text { months } \\
(31 \%) \\
6 \text { months } \\
(30 \%)\end{array}$ & $\begin{array}{l}\text { Improvements between } 2.5 \text { and } 5 \\
\text { months, and between } 5 \text { and } 9 \text { months }\end{array}$ & $\begin{array}{l}\text { Hospital admission and female gender } \\
\text { were predictive for long-term HRQL }\end{array}$ \\
\hline $\begin{array}{l}\text { Polinder, 2007, } \\
\text { Netherlands } \\
\text { [37] }\end{array}$ & $\begin{array}{l}\text { ED and/or } \\
\text { admitted } \\
\text { Age: }>14 \\
(\mathrm{n}=8564)\end{array}$ & $\begin{array}{l}\text { EQ-5D } \\
\text { (Self-completed) }\end{array}$ & $\begin{array}{l}2.5 \text { month } \\
(37 \%) \\
5 \text { months } \\
(28 \%) \\
9 \text { months } \\
(27 \%) \\
24 \text { months } \\
(21 \%)\end{array}$ & $\begin{array}{l}\text { Improvement among non admitted } \\
\text { patients until } 5 \text { months, and among } \\
\text { admitted patients until } 24 \text { months }\end{array}$ & $\begin{array}{l}\text { Hospitalization, age and sex (females), } \\
\text { type of injury and comorbidity were } \\
\text { significant predictors of poor functioning } \\
\text { in the long term }\end{array}$ \\
\hline $\begin{array}{l}\text { Vasquez, 1996, } \\
\text { Spain [56] }\end{array}$ & $\begin{array}{l}\text { Admitted } \\
\text { ICU patients } \\
(\mathrm{n}=351)\end{array}$ & $\begin{array}{l}\text { GOS } \\
\text { (Self-completed) }\end{array}$ & $\begin{array}{l}\text { Admission } \\
1 \text { year } \\
2 \text { year (\% } \\
\text { not } \\
\text { available) }\end{array}$ & $\begin{array}{l}\text { Quality of life improved the first year } \\
\text { and between } 1 \text { and } 2 \text { years, but after } \\
2 \text { years still below pre-admission } \\
\text { summary scores }\end{array}$ & $\begin{array}{l}\text { Long-term HRQL was associated with age, } \\
\text { injury severity, and previous quality of life }\end{array}$ \\
\hline $\begin{array}{l}\text { Hetherington, } \\
\text { 1995, UK [28] }\end{array}$ & $\begin{array}{l}\text { Trauma } \\
\text { helicopter } \\
\text { patients } \\
(\mathrm{n}=100)\end{array}$ & $\begin{array}{l}\text { FIM } \\
\text { (Face to face } \\
\text { interviews) }\end{array}$ & $\begin{array}{l}\text { Acute } \\
3 \text { months } \\
6 \text { months } \\
(93 \%)\end{array}$ & $\begin{array}{l}\text { Improvements in mobility and self care } \\
\text { between } 3 \text { and } 6 \text { months }\end{array}$ & $\begin{array}{l}\text { HRQL associated with length of hospital } \\
\text { stay }\end{array}$ \\
\hline $\begin{array}{l}\text { Gofin, 1997, } \\
\text { Israel [57] }\end{array}$ & $\begin{array}{l}\text { Age 4-17 } \\
(n=281)\end{array}$ & $\begin{array}{l}\text { ICIDH } \\
\text { (Telephone } \\
\text { parent } \\
\text { interviews) }\end{array}$ & $\begin{array}{l}\text { Immediately } \\
6 \text { months } \\
(85 \%)\end{array}$ & $\begin{array}{l}\text { Improvements until } 6 \text { months after } \\
\text { injury }\end{array}$ & HRQL is positively associated with ISS \\
\hline $\begin{array}{l}\text { Gofin, 1995, } \\
\text { Israel [58] }\end{array}$ & $\begin{array}{l}\text { Age } 0-17 \\
(n=432)\end{array}$ & $\begin{array}{l}\text { ICIDH } \\
\text { (Telephone } \\
\text { parent } \\
\text { interviews) }\end{array}$ & $\begin{array}{l}\text { Immediately } \\
6 \text { months } \\
(85 \%)\end{array}$ & $\begin{array}{l}\text { Improvements until } 6 \text { months after } \\
\text { injury }\end{array}$ & $\begin{array}{l}\text { HRQL associated with child's age and } \\
\text { parental proxy age }\end{array}$ \\
\hline $\begin{array}{l}\text { Holbrook, } \\
\text { 1998, US [14] }\end{array}$ & $\begin{array}{l}>24 \text { hours } \\
\text { admitted in } \\
\text { trauma } \\
\text { center } \\
\text { Age } 18+ \\
\text { GCS }>11 \\
(n=1048)\end{array}$ & $\begin{array}{l}\text { QWB-scale } \\
\text { (Face to face } \\
\text { interviews) }\end{array}$ & $\begin{array}{l}\text { Pre-injury } \\
\text { Discharge } \\
6 \text { months } \\
(79 \%)\end{array}$ & $\begin{array}{l}\text { Significant degree of functional } \\
\text { limitations at discharge compared to } \\
\text { pre-injury scores. }\end{array}$ & $\begin{array}{l}\text { Post-injury depression, PTSD, serious } \\
\text { extremity injury, and length of stay were } \\
\text { significant predictors of HRQL }\end{array}$ \\
\hline $\begin{array}{l}\text { Holbrook, } \\
\text { 1999, US [15] }\end{array}$ & $\begin{array}{l}>24 \text { hours } \\
\text { admitted in } \\
\text { trauma } \\
\text { center } \\
\text { Age } 18+ \\
\text { GCS }>11 \\
(\mathrm{n}=1048)\end{array}$ & $\begin{array}{l}\text { QWB-scale } \\
\text { (Face to face } \\
\text { interviews) }\end{array}$ & $\begin{array}{l}\text { Pre-injury } \\
\text { Discharge } \\
\text { (79\%) } \\
12 \text { months } \\
(79 \%) \\
18 \text { months } \\
(74 \%)\end{array}$ & $\begin{array}{l}\text { Improvement between discharge and } \\
12 \text { months, but no improvements } \\
\text { between } 12 \text { and } 18 \text { months. }\end{array}$ & $\begin{array}{l}\text { Post-injury depression, PTSD, serious } \\
\text { extremity injury, and intensive care unit } \\
\text { days were significant independent } \\
\text { predictors of HRQL }\end{array}$ \\
\hline $\begin{array}{l}\text { Holbrook, } \\
\text { 2004, US [16] }\end{array}$ & $\begin{array}{l}>24 \text { hours } \\
\text { admitted in } \\
\text { trauma } \\
\text { center } \\
\text { Age } 18+ \\
\text { GCS }>11 \\
(n=1048)\end{array}$ & $\begin{array}{l}\text { QWB-scale } \\
\text { (Face to face } \\
\text { interviews) }\end{array}$ & $\begin{array}{l}\text { Discharge } \\
(79 \%) \\
6 \text { months } \\
(79 \%) \\
12 \text { months } \\
(74 \%) \\
18 \text { months } \\
(74 \%)\end{array}$ & $\begin{array}{l}\text { Improvement between } 6 \text { and } \\
12 \text { months }\end{array}$ & $\begin{array}{l}\text { Gender (women) was a significant } \\
\text { independent predictors of HRQL at all } \\
\text { follow-up time points }\end{array}$ \\
\hline
\end{tabular}


Table 1 Study characteristics and methodological aspects of follow-up studies measuring health-related quality of life of injury patients (in order of nr of HRQL instruments used - bold author names are studies of children) (Continued)

\begin{tabular}{|c|c|c|c|c|c|}
\hline $\begin{array}{l}\text { Gabbe, 2007, } \\
\text { Australia [31] }\end{array}$ & $\begin{array}{l}\text { Admitted } \\
\text { Age } 18-64 \\
(n=1033)\end{array}$ & $\begin{array}{l}\text { SF-12 } \\
\text { (Telephone } \\
\text { interviews) }\end{array}$ & $\begin{array}{l}\text { Pre-injury } \\
12 \text { months } \\
(69 \%)\end{array}$ & $\begin{array}{l}\text { After } 12 \text { months patients were not } \\
\text { returned to their pre-injury status }\end{array}$ & $\begin{array}{l}\text { Compensable patients were more likely } \\
\text { than non-compensable patients to report } \\
\text { moderate to severe HRQL }\end{array}$ \\
\hline $\begin{array}{l}\text { Brenneman, } \\
\text { 1997, Canada } \\
\text { [59] }\end{array}$ & $\begin{array}{l}\text { Admitted } \\
\text { ISS }>10 \\
(\mathrm{~N}=195)\end{array}$ & $\begin{array}{l}\text { SF-36 } \\
\text { (Telephone } \\
\text { interviews) }\end{array}$ & $\begin{array}{l}\text { Discharge } \\
(56 \%) \\
12 \text { months } \\
(44 \%)\end{array}$ & $\begin{array}{l}\text { Improvements between discharge and } \\
12 \text { months }\end{array}$ & $\begin{array}{l}\text { Better scores on } 7 \text { dimensions of the } \\
\text { SF-36 for patients who returned to work }\end{array}$ \\
\hline $\begin{array}{l}\text { Michaels, } \\
\text { 1999, US [20] }\end{array}$ & $\begin{array}{l}\text { Admitted to } \\
\text { trauma } \\
\text { centre } \\
\text { Age } 18+ \\
(n=247)\end{array}$ & $\begin{array}{l}\text { SF-36 } \\
\text { (Self-completed) }\end{array}$ & $\begin{array}{l}\text { Admission } \\
(100 \%) \\
6 \text { months } \\
(75 \%) \\
12 \text { months } \\
(51 \%)\end{array}$ & $\begin{array}{l}\text { Improvements between baseline and } \\
6 \text { months, and between } 6 \text { and } \\
12 \text { months }\end{array}$ & $\begin{array}{l}\text { Mental health (PTSD) is an independent } \\
\text { predictor of HRQL }\end{array}$ \\
\hline $\begin{array}{l}\text { Kopjar, 1996, } \\
\text { Norway [60] }\end{array}$ & $\begin{array}{l}\text { Treated for } \\
\text { injury } \\
\text { Age 16-78 } \\
(n=775)\end{array}$ & $\begin{array}{l}\text { SF-36 } \\
\text { (Self-completed) }\end{array}$ & $\begin{array}{l}6-10 \text { weeks } \\
(61 \%) \\
24-28 \\
\text { weeks } \\
(63 \%)\end{array}$ & $\begin{array}{l}\text { Improvements between } 2 \text { and } \\
\text { six months }\end{array}$ & HRQL associated with activity restrictions \\
\hline $\begin{array}{l}\text { Macpherson, } \\
\text { 2003, } \\
\text { Canada [39] }\end{array}$ & $\begin{array}{l}\text { Hospital } \\
\text { inpatients } \\
\text { Age } 2-15 \\
\text { ISS }>12 \\
(\mathrm{n}=489)\end{array}$ & $\begin{array}{l}\text { WeeFIM } \\
\text { (Telephone } \\
\text { interviews) }\end{array}$ & $\begin{array}{l}\text { Discharge } \\
6 \text { months } \\
(73 \%)\end{array}$ & $\begin{array}{l}\text { Improvement between discharge and } \\
6 \text { months }\end{array}$ & $\begin{array}{l}\text { Injury mechanism is an independent } \\
\text { predictor of } \mathrm{HRQL}\end{array}$ \\
\hline $\begin{array}{l}\text { Aitken, 2002, } \\
\text { US [11] }\end{array}$ & $\begin{array}{l}\text { Admitted to } \\
\text { children's } \\
\text { hospital } \\
\text { Age } 3-18 \\
\text { ISS > } 3 \\
(\mathrm{n}=310)\end{array}$ & $\begin{array}{l}\text { CHQ PF-50, } \\
\text { WeeFIM } \\
\text { (Parent } \\
\text { interview, child } \\
\text { Self-completed) }\end{array}$ & $\begin{array}{l}\text { Discharge } \\
63 \%) \\
1 \text { month } \\
(56 \%) \\
6 \text { months } \\
(45 \%)\end{array}$ & Improvements between 1 and 6 months & $\begin{array}{l}\text { HRQL associated with level of injury } \\
\text { severity }\end{array}$ \\
\hline $\begin{array}{l}\text { Winthrop, } \\
2005 \text {, US [21] }\end{array}$ & $\begin{array}{l}\text { Admitted } \\
\text { Age } 1-18 \\
\text { ISS }>8 \\
(n=180)\end{array}$ & $\begin{array}{l}\mathrm{CHQ}, \mathrm{FIM} \\
\text { (Face-to-face } \\
\text { interviews) }\end{array}$ & $\begin{array}{l}\text { Discharge } \\
(90 \%) \\
1 \text { month } \\
6 \text { months } \\
(87 \%) \\
12 \text { months }\end{array}$ & $\begin{array}{l}\text { Improvements between baseline and } \\
1 \text { month, and between } 1 \text { and } 6 \text { months }\end{array}$ & HRQL associated with injury severity \\
\hline $\begin{array}{l}\text { Baldry } \\
\text { Currens, 2000, } \\
\text { UK }[24,25]\end{array}$ & $\begin{array}{l}\text { Survivors of } \\
\text { major } \\
\text { trauma } \\
\text { Admitted }>3 \\
\text { days } \\
\text { Age } 5+ \\
(\mathrm{n}=251)\end{array}$ & $\begin{array}{l}\text { FIM, GOS } \\
\text { (Telephone } \\
\text { interviews) }\end{array}$ & $\begin{array}{l}3 \text { months } \\
(80 \%) \\
6 \text { months } \\
12 \text { months } \\
>24 \\
\text { months }\end{array}$ & $\begin{array}{l}\text { Improvements between } 3 \text { and } 6 \text { months } \\
\text { No further improvement between } 6 \\
\text { months and } 1 \text { year }\end{array}$ & $\begin{array}{l}\text { HRQL associated with major vs. minor } \\
\text { injury and body region injured }\end{array}$ \\
\hline $\begin{array}{l}\text { Gillen, 2004, } \\
\text { US [13] }\end{array}$ & $\begin{array}{l}\text { Age } 20+ \\
(n=114)\end{array}$ & $\begin{array}{l}\text { SF-36, HAQ } \\
\text { (Telephone } \\
\text { interviews) }\end{array}$ & $\begin{array}{l}1 \text { week } \\
2 \text { weeks } \\
1 \text { month } \\
3 \text { months } \\
(79 \%)\end{array}$ & $\begin{array}{l}\text { Improvements between } 1 \text { week and } 2 \\
\text { weeks, between } 2 \text { weeks and } 1 \text { month, } \\
\text { and between } 1 \text { and } 3 \text { months. }\end{array}$ & $\begin{array}{l}\text { The SF-36 and the HAQ were responsive } \\
\text { to clinical changes in varying conditions } \\
\text { and the SF- } 36 \text { was sensitive to changes in } \\
\text { traumatic injuries. }\end{array}$ \\
\hline $\begin{array}{l}\text { Kiely, 2006, } \\
\text { US [18] }\end{array}$ & $\begin{array}{l}\text { Age } 18-55 \\
\text { ISS }>8 \text { and } \\
\text { all patients } \\
\text { with age } 55 \\
+ \\
(\mathrm{n}=312)\end{array}$ & $\begin{array}{l}\text { SF-36, FIM } \\
\text { (Telephone } \\
\text { interview or self- } \\
\text { completed) }\end{array}$ & $\begin{array}{l}1 \text { month } \\
(63 \%) \\
6 \text { months } \\
(39 \%)\end{array}$ & $\begin{array}{l}\text { Improvements between } 1 \text { and } 6 \text { months } \\
\text { post injury }\end{array}$ & $\begin{array}{l}\text { Functional status, PTSD, social support, } \\
\text { and depression were predictors of HRQL }\end{array}$ \\
\hline $\begin{array}{l}\text { Soberg, 2007, } \\
\text { Norway [43] }\end{array}$ & $\begin{array}{l}\text { Admitted to } \\
\text { trauma } \\
\text { centre } \\
\text { ISS > } 15 \\
\text { Age } 18-67 \\
(\mathrm{n}=169)\end{array}$ & $\begin{array}{l}\text { SF-36, } \\
\text { WHODASH II } \\
\text { (Self-completed) }\end{array}$ & $\begin{array}{l}6 \text { weeks } \\
(62 \%) \\
1 \text { year } \\
(61 \%) \\
2 \text { years } \\
(60 \%)\end{array}$ & $\begin{array}{l}\text { Improvements, except for mental and } \\
\text { general health between } 6 \text { wk and } 1 \text { year. } \\
\text { Between } 1 \text { and } 2 \text { years almost no } \\
\text { improvements. }\end{array}$ & $\begin{array}{l}\text { Profession, injury severity, pain, and } \\
\text { physical, cognitive, and social functioning } \\
\text { made independent contributions to } \\
\text { WHODAS II } 2 \text { years after injury }\end{array}$ \\
\hline
\end{tabular}


Table 1 Study characteristics and methodological aspects of follow-up studies measuring health-related quality of life of injury patients (in order of nr of HRQL instruments used - bold author names are studies of children) (Continued)

\begin{tabular}{|c|c|c|c|c|c|}
\hline $\begin{array}{l}\text { Evanoff, 2002, } \\
\text { US [12] }\end{array}$ & $\begin{array}{l}\text { Workers }>5 \\
\text { days } \\
\text { workdays } \\
\text { lost } \\
(\mathrm{n}=934)\end{array}$ & $\begin{array}{l}\text { SF-36, SF-12, } \\
\text { DASH short } \\
\text { version } \\
\text { (Face to face } \\
\text { interviews) }\end{array}$ & $\begin{array}{l}\text { Baseline } \\
(33 \%) \\
6 \text { months } \\
(27 \%)\end{array}$ & $\begin{array}{l}\text { Improvement over } 6 \text { months, after } \\
6 \text { months continuing } \mathrm{HRQL}\end{array}$ & No \\
\hline $\begin{array}{l}\text { Watson, 2005, } \\
\text { Australia [33] }\end{array}$ & $\begin{array}{l}\text { Admitted } \\
\text { Age 18-74 } \\
(n=221)\end{array}$ & $\begin{array}{l}\text { SF-36, AQol, } \\
\text { SF-6D } \\
\text { (Face-to-face } \\
\text { interviews) }\end{array}$ & $\begin{array}{l}\text { Pre-injury } \\
1,6,12 \\
\text { weeks } \\
6 \text { months } \\
12 \text { months } \\
(84 \%)\end{array}$ & $\begin{array}{l}\text { Significant improvement of functional } \\
\text { outcome till } 6 \text { months; no significant } \\
\text { difference in summary scores at } 6 \text { and } \\
12 \text { months post-injury }\end{array}$ & $\begin{array}{l}\text { The AQoL showed good discrimination } \\
\text { between groups for type of injury, body } \\
\text { region injured and severity of injury }\end{array}$ \\
\hline $\begin{array}{l}\text { Watson, 2007, } \\
\text { Australia [33] }\end{array}$ & $\begin{array}{l}\text { Admitted } \\
\text { Age 18-74 } \\
(n=186)\end{array}$ & $\begin{array}{l}\text { SF-36, AQol, } \\
\text { SF-6D } \\
\text { (Face-to-face } \\
\text { interviews) }\end{array}$ & $\begin{array}{l}\text { Pre-injury } \\
12 \text { months } \\
(88 \%)\end{array}$ & $\begin{array}{l}\text { No difference between summary scores } \\
\text { at baseline and } 12 \text { months after injury } \\
\text { for patients that completely recovered }\end{array}$ & $\begin{array}{l}\text { Gender, age, working status were } \\
\text { predictors for complete recovery after one } \\
\text { year }\end{array}$ \\
\hline $\begin{array}{l}\text { Gabbe, 2008, } \\
\text { Australia [32] }\end{array}$ & $\begin{array}{l}\text { Age } 15-80 \\
\text { ISS }>15 \\
(n=243)\end{array}$ & $\begin{array}{l}\text { FIM, Modified } \\
\text { FIM, GOS, GOS-E } \\
\text { (Telephone } \\
\text { interviews with } \\
\text { participants or } \\
\text { care provider) }\end{array}$ & $\begin{array}{l}\text { Discharge } \\
6 \text { months } \\
(97 \%)\end{array}$ & $\begin{array}{l}\text { Improvement between discharge and } \\
6 \text { months, except for the cognition } \\
\text { component of the FIM }\end{array}$ & $\begin{array}{l}\text { HRQL associated with discharge } \\
\text { destination and head injury vs. no head } \\
\text { injury }\end{array}$ \\
\hline $\begin{array}{l}\text { Sutherland, } \\
\text { 2005, UK [29] }\end{array}$ & $\begin{array}{l}\text { Admitted } \\
\text { Age } 16-70 \\
(n=200)\end{array}$ & $\begin{array}{l}\text { SF-36, SF-6D, } \\
\text { MFA, GHQ } \\
\text { (Self-completed) }\end{array}$ & $\begin{array}{l}2 \text { months } \\
(79 \%) \\
6 \text { months } \\
(75 \%)\end{array}$ & $\begin{array}{l}\text { No improvement between } 2 \text { and } \\
6 \text { months }\end{array}$ & No \\
\hline
\end{tabular}

AMA-guides = American Medical Association guides; BDS = Bull Disability Scale; CFS = Cognitive Function Scale; CHQ PF-50 = Parent Completed version of the $\mathrm{CHQ} ; \mathrm{CHQ}=$ Child Health Questionnaire; EQ5 D = European Quality of life instrument-5 dimensions; FIM = Functional Independence Measure; GHQ = General Health Questionnaire; GOS = Glasgow Outcome Scale; HAQ = Health Assessment Questionnaire; HOBQ = Health Outcomes Burn Questionnaire for Children; ICIDH2 = 25 item scale for measuring functional outcome by the International Classification of Impairments Disabilities and Handicaps; MFA = Musculoskeletal Functional Assessment; NHP = Nottingham Health Profile; OPCS = Office of Population Census and Surveys national survey of disability in Great Britain; QOL = Satisfaction with Quality of Life instrument; QWB = Quality of Well Being scale; RDS = Rosser Disease Score; RTW = Return To pre-injury Work status; SF-6D = Medical Outcome Study Short Form-6 dimensions; SF-12 = Medical Outcome Study Short Form-12 items; SF-36 = Medical Outcome Study Short form-36 items; SIP = Sickness Impact Profile; TOP = Trauma Outcome Profile; WeeFIM = Pediatric version of the FIM; WODASII = World Health Organization Disability Assessment Schedule version II; WHOQOL-BREF = short version of the World Health Organization Quality of life.

There were twelve studies that reported HRQL utility scores. Table 3 shows that the injury populations differed considerably. All studies reported measurable recovery in the first year after injury. Among the severely injury patients there was some evidence of further improvement in the second year. Figure 3 provides an overview of the studies that reported HRQL summary scores over time in the 12 months following discharge among patients aged 15 or older. Overall, HRQL improves in the first year after discharge, although the large variation in HRQL instruments, study population and time points at which HRQL was assessed impedes comparison of HRQL summary scores between studies.

\section{Discussion}

This systematic review aimed to provide greater insight into the measurement of functional outcome and recovery patterns of general injury populations in studies using a generic health state measure. There was considerable methodological variation between studies, including different settings, mixture of participants, instruments, and follow-up periods and timings of assessment. Among available generic instruments, the SF-36, FIM, GOS and EQ-5D have been most frequently used. Studies of functional outcome of the general injury population are still uncommon and generally not comparable, preventing an in-depth understanding of the HRQL experiences of injured persons. Evidence from our review lends support to the need for guidelines for the conduct of follow-up studies measuring injury-related disability.

Longitudinal studies with multiple time points measuring outcomes, and incorporating a retrospective assessment of the pre-injury situation are needed to produce valid estimates of injury-related morbidity and disability. Studies with this design provide insight into the course of recovery over time and quantify the longerterm functional consequences of injuries. There is still a lack of consensus on preferred HRQL instruments and study designs given the wide variety of different approaches that are used by the articles included in this review. This variability prevents meta-analyses necessary to refine quantification of the impact of injury on population health over time.

Almost no papers provided a description of the evaluation of the instruments used against widely accepted 
Table 2 Study characteristics and methodological aspects of studies measuring health-related quality of life of injury patients at one time point (in order of $\mathrm{nr}$ of HRQL instruments used - bold author names are studies of children)

\begin{tabular}{|c|c|c|c|c|}
\hline $\begin{array}{l}\text { Author, year, } \\
\text { country }\end{array}$ & Study population & $\begin{array}{l}\text { HRQL instrument } \\
\text { (mode of } \\
\text { administration) }\end{array}$ & $\begin{array}{l}\text { Follow up } \\
\text { (response } \\
\text { rates) }\end{array}$ & Predictors for HRQL \\
\hline $\begin{array}{l}\text { Braithwaite, 1998, } \\
\text { UK [26] }\end{array}$ & $\begin{array}{l}\text { Severe injuries } \\
\text { ISS }>15 \\
\text { Age } 15+ \\
(\mathrm{n}=212)\end{array}$ & $\begin{array}{l}\text { BDS } \\
\text { (Face to face interview) }\end{array}$ & 5 years $(75 \%)$ & HRQL associated with body region injured \\
\hline $\begin{array}{l}\text { Korosec, 2006, } \\
\text { Slovenia [61] }\end{array}$ & $\begin{array}{l}\text { ICU patients } \\
(\mathrm{n}=98)\end{array}$ & $\begin{array}{l}\text { EQ5D } \\
\text { (Telephone interview) }\end{array}$ & $\begin{array}{l}2 \text { years (\% not } \\
\text { available) }\end{array}$ & No \\
\hline $\begin{array}{l}\text { Aitken, 1999, US } \\
\text { and Canada [22] }\end{array}$ & $\begin{array}{l}\text { Hospitalized } \\
\text { Age 7-18 } \\
(n=13649) \\
\end{array}$ & $\begin{array}{l}\text { FIM } \\
\text { (Filled out by doctors) }\end{array}$ & Discharge (80\%) & $\begin{array}{l}\text { Functional outcome associated with type of injury. } \\
\text { Lower extremity fractures caused more limitations at } \\
\text { discharge as compared to other injuries }\end{array}$ \\
\hline $\begin{array}{l}\text { Evans, 2003, UK } \\
{[27]}\end{array}$ & $\begin{array}{l}\text { Age } 11-24 \\
\text { ISS }>15 \\
(n=125)\end{array}$ & $\begin{array}{l}\text { OPCS } \\
\text { (Face to face interview) }\end{array}$ & 5 years $(87 \%)$ & Not measured \\
\hline $\begin{array}{l}\text { Holtslag, 2007, } \\
\text { Netherlands [34] }\end{array}$ & $\begin{array}{l}\text { Age } 16+ \\
\text { ISS }>15 \\
(n=359)\end{array}$ & $\begin{array}{l}\text { SIP } \\
\text { (Self-completed) }\end{array}$ & $\begin{array}{l}\text { Between } 12-18 \\
\text { months }(93 \%)\end{array}$ & $\begin{array}{l}\text { Age, comorbidity, and type of injury were predictors of } \\
\text { HRQL }\end{array}$ \\
\hline $\begin{array}{l}\text { MacKenzie, 2002, } \\
\text { US [19] }\end{array}$ & $\begin{array}{l}\text { Admitted }>72 \text { hours or } \\
\text { to ICU } \\
\text { Aga } 18-59 \text { years } \\
(\mathrm{n}=1587)\end{array}$ & $\begin{array}{l}\text { SF-36 + cognitive } \\
\text { functioning scale } \\
\text { (Face to face interview) }\end{array}$ & 12 months (78\%) & $\begin{array}{l}\text { Cognitive functioning and head injury were predictors of } \\
\text { HRQL }\end{array}$ \\
\hline $\begin{array}{l}\text { Alves, 2009, } \\
\text { Brazil [62] }\end{array}$ & $\begin{array}{l}\text { ED and admitted }>24 \\
\text { hours } \\
\text { Age } 16-65 \\
\text { GCS }>9 \text { and ISS }>5 \\
(n=35)\end{array}$ & $\begin{array}{l}\text { WHOQOL-BREF } \\
\text { (face-to-face interview) }\end{array}$ & 6 months (88\%) & $\begin{array}{l}\text { Hospitalization, age, and sex were predictors for } \\
\text { functional impairment in the physical domain }\end{array}$ \\
\hline Airey, 2001, UK [23] & $\begin{array}{l}\text { Admitted survivors of } \\
\text { major trauma } \\
\text { ISS }>15 \\
(\mathrm{n}=367)\end{array}$ & $\begin{array}{l}\text { SF-36, OPCS } \\
\text { (Face to face } \\
\text { interviews) }\end{array}$ & 5 years $(84 \%)$ & $\begin{array}{l}\text { HRQL associated with injury severity and general health } \\
\text { perception }\end{array}$ \\
\hline $\begin{array}{l}\text { Pirente, 2001, } \\
\text { Germany [63] }\end{array}$ & $\begin{array}{l}\text { Admitted and 'severely } \\
\text { injured' } \\
(n=56)\end{array}$ & $\begin{array}{l}\text { SF-36, TOP } \\
\text { (Telephone interview } \\
\text { or self-completed) }\end{array}$ & 12 months (77\%) & $\begin{array}{l}\text { HRQL among trauma patients higher than control group } \\
\text { on al SF-36 dimensions (no injury) }\end{array}$ \\
\hline $\begin{array}{l}\text { Holtslag, 2008, } \\
\text { Netherlands [64] }\end{array}$ & $\begin{array}{l}\text { Age } 16+ \\
\text { ISS }>15 \\
(n=359)\end{array}$ & $\begin{array}{l}\text { GOS, EQ-5D } \\
\text { (self-completed) }\end{array}$ & $\begin{array}{l}\text { Between } 12-18 \\
\text { months }(93 \%)\end{array}$ & $\begin{array}{l}\text { Injury type and comorbidity were significantly associated } \\
\text { with HRQL }\end{array}$ \\
\hline $\begin{array}{l}\text { Janssens, 2009, } \\
\text { Netherlands [38] }\end{array}$ & $\begin{array}{l}\text { Admitted to trauma } \\
\text { center } \\
\mathrm{Ag}<16 \\
\text { ISS }>15 \\
(\mathrm{n}=40)\end{array}$ & $\begin{array}{l}\text { GOS and GOSE, AMA } \\
\text { guides } \\
\text { (Self-completed and } \\
\text { face-to-face interview) }\end{array}$ & 7 years $(70 \%)$ & $\begin{array}{l}\text { Good discrimination could be made between } \\
\text { respondents with different levels of functional } \\
\text { impairment }\end{array}$ \\
\hline $\begin{array}{l}\text { Dimopoulou, 2004, } \\
\text { Greece [65] }\end{array}$ & $\begin{array}{l}\text { Admitted multiple } \\
\text { trauma patients } \\
(n=117)\end{array}$ & $\begin{array}{l}\text { GOS, NHP, RDS } \\
\text { (Telephone interview) }\end{array}$ & 12 months (74\%) & $\begin{array}{l}\text { HRQL associated with injury severity } \\
\text { ISS and severe head trauma were independent predictors } \\
\text { of poor HRQL }\end{array}$ \\
\hline $\begin{array}{l}\text { Keyes, 2001, } \\
\text { US [17] }\end{array}$ & $\begin{array}{l}\text { Workers with }>3 \text { days } \\
\text { work loss }(n=402)\end{array}$ & $\begin{array}{l}\text { SF-36, HAQ, QOL } \\
\text { (Telephone interview } \\
\text { or self-completed) }\end{array}$ & 2 years (93\%) & No \\
\hline $\begin{array}{l}\text { Stalp, 2001, } \\
\text { Germany [66] }\end{array}$ & $\begin{array}{l}\text { Admitted } \\
\text { ISS }>15 \\
(\mathrm{n}=150)\end{array}$ & $\begin{array}{l}\text { SF-12, FIM, GOS, MFA } \\
\text { (Self-completed) }\end{array}$ & 24 months (81\%) & HRQL associated with body region injured \\
\hline $\begin{array}{l}\text { Stalp, 2002, } \\
\text { Germany [67] }\end{array}$ & $\begin{array}{l}\text { Admitted } \\
\text { ISS }>15 \\
(\mathrm{n}=312)\end{array}$ & $\begin{array}{l}\text { SF-12, FIM, GOS, MFA } \\
\text { (Self-completed) }\end{array}$ & 24 months (81\%) & $\begin{array}{l}\text { HRQL associated with body region injured } \\
\text { (highest for lower extremity injury) }\end{array}$ \\
\hline
\end{tabular}

AMA-guides = American Medical Association guides; BDS = Bull Disability Scale; CFS = Cognitive Function Scale; CHQ PF-50 = Parent Completed version of the $\mathrm{CHQ} ; \mathrm{CHQ}=$ Child Health Questionnaire; EQ5 D = European Quality of life instrument-5 dimensions; FIM = Functional Independence Measure; GHQ = General Health Questionnaire; GOS = Glasgow Outcome Scale; HAQ = Health Assessment Questionnaire; HOBQ = Health Outcomes Burn Questionnaire for Children; ICIDH2 = 25 item scale for measuring functional outcome by the International Classification of Impairments Disabilities and Handicaps; MFA = Musculoskeletal Functional Assessment; NHP = Nottingham Health Profile; OPCS = Office of Population Census and Surveys national survey of disability in Great Britain; QOL = Satisfaction with Quality of Life instrument; QWB = Quality of Well Being scale; RDS = Rosser Disease Score; RTW = Return To pre-injury Work status; SF-6D = Medical Outcome Study Short Form-6 dimensions; SF-12 = Medical Outcome Study Short Form-12 items; SF-36 = Medical Outcome Study Short form-36 items; SIP = Sickness Impact Profile; TOP = Trauma Outcome Profile; WeeFIM = Pediatric version of the FIM; WODASII = World Health Organization Disability Assessment Schedule version II; WHOQOL-BREF = short version of the World Health Organization Quality of life. 


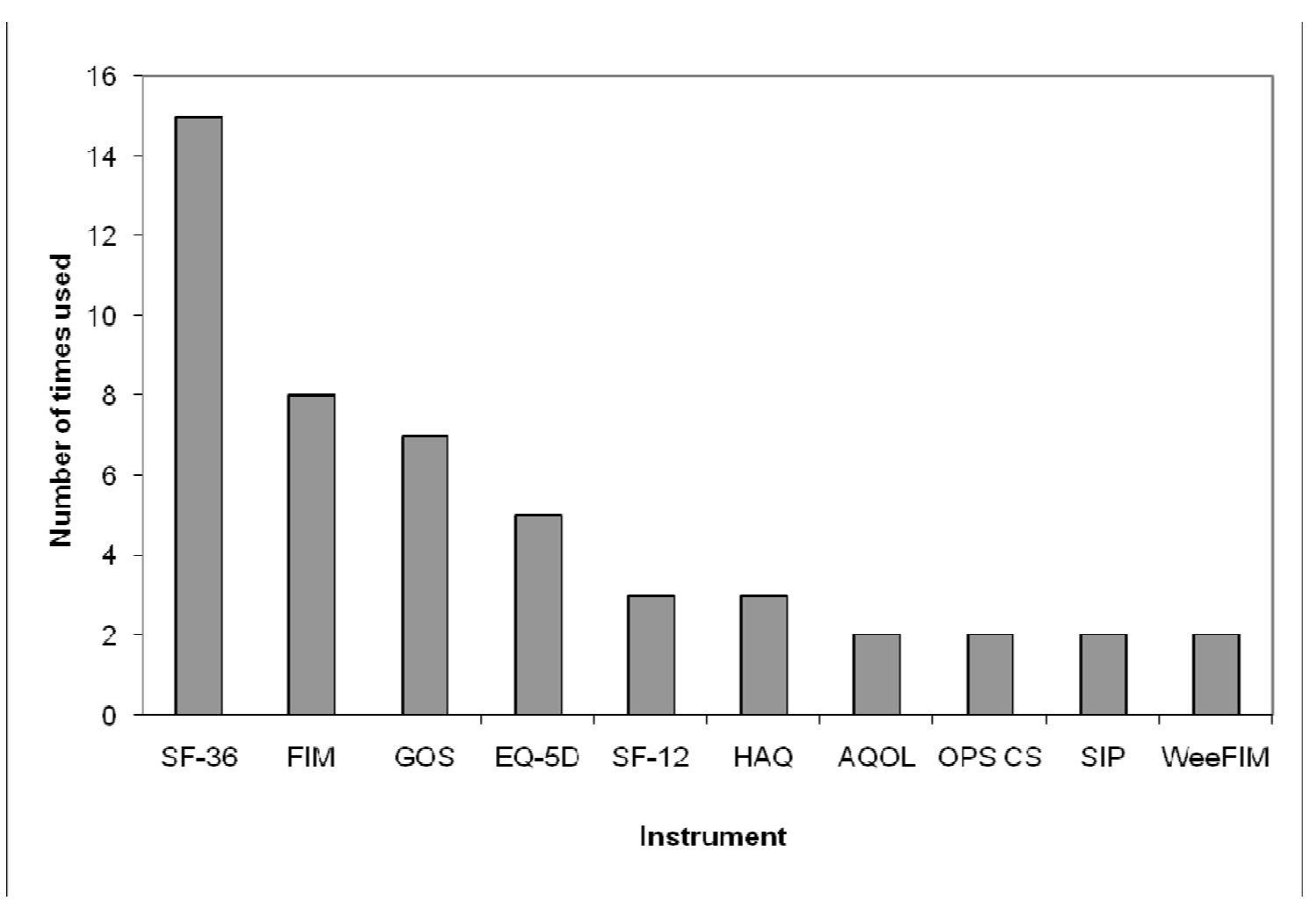

Footnote: The figures are based on number of cohorts instead of number of studies. Instruments that were used once were not displayed in the figure.

Figure 1 Instruments used in patient follow-up studies for assessing injury-related disability. SF-36 = Medical Outcome Study Short form36 items; FIM = Functional Independence Measure; GOS = Glasgow Outcome Scale; EQ5 D = European Quality of life instrument-5 dimensions; SF-12 = Medical Outcome Study Short Form-12 items; SF-6D = Medical Outcome Study Short Form-6 dimensions; QWB = Quality of Well Being scale; $\mathrm{HAQ}=$ Health Assessment Questionnaire; $\mathrm{AQOL}=$ Assessment of Quality of Life instrument; OPCS = Office of Population Census and Surveys national survey of disability in Great Britain; ICIDH $=25$ item scale for measuring functional outcome by the International Classification of Impairments Disabilities and Handicaps; $\mathrm{CHQ}=$ Child Health Questionnaire.

criteria with in the field: data quality, reliability, validity and responsiveness (e.g. the COSMIN checklist [44]). Preferably, instruments should only be widely applied within the injury field if there is acceptable evidence for these measurement criteria in the population of interest. Empirical head-to-head comparisons of different HRQL measures are needed to obtain more insight into the strengths and limitations of the multi-attribute utility measures (MAUI) to estimate utility losses in injury populations. Such head-to-head comparisons have so far been lacking. Several of the authors have recently published a paper comparing the Health Utility Index (HUI) mark 2 and 3 and the EQ-5D in 'all injury patients' [45]. However, to maximise the utility of the available sparse data there is a need for studies which develop translational or bridging metrics for the different instruments which would then allow data to be combined in metaanalyses.

The EuroSafe Group has developed guidelines which advise the use of a combination of the EQ-5D and the
HUI, with assessments at $1,2,4$ and 12 months after injury [1]. Few studies, including those published after 2007, satisfy the guidelines. Only five studies used the EQ-5D, and none was found that used the HUI. The guidance recommended the use of two instruments to measure functional outcome, but half of the studies only used one instrument. With the exception of the twelve month measurement few recommended time points were assessed. However, most of the published studies were designed before publication of the guidelines in 2007.

Different HRQL instruments assess different dimensions of health, which make comparisons of study outcomes difficult. Polinder et al. showed that the HUI and EQ-5D resulted in significantly different utilities for similar health states for a general injury population [45]. These differences have the undesirable effect that the distinct instruments yield different utilities for similar health states. Clinicians and researchers should be aware of these differences between the HRQL instruments. 


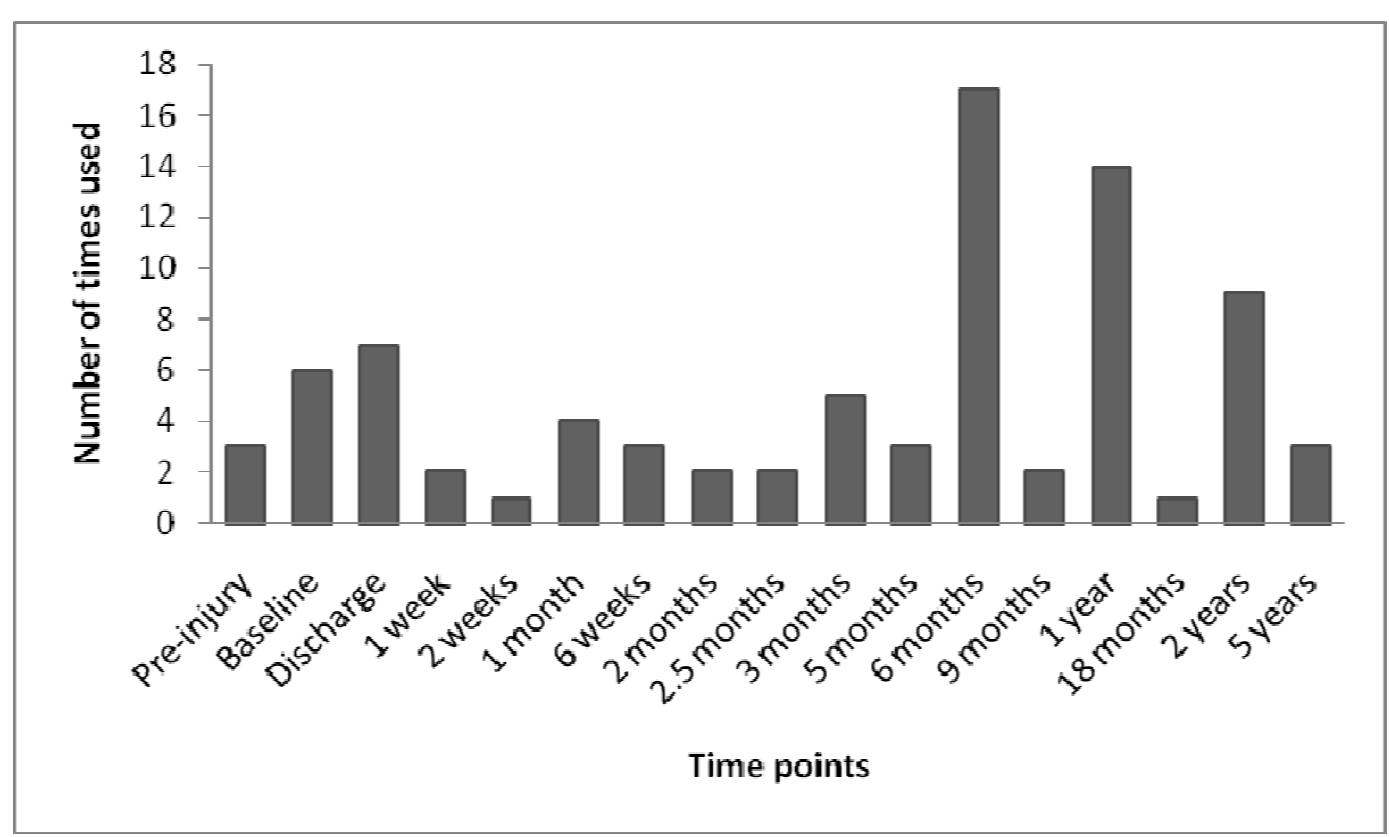

Footnote: The figures are based on number of cohorts instead of number of studies. Baseline $=$ acute (immediately after the injury) or during admission; discharge=at hospital discharge.

Figure 2 Time points at which injury-related disability was assessed.

It is remarkable that in the 41 papers reviewed 24 different HRQL instruments were used. The use of so many different HRQL instruments might indicate that none of the instruments seems to incorporate all the attributes that one would like to, or that there is uncertainty about which instrument is best to use. Decisions regarding which HRQL measure to use will be influenced by a range of factors. For example, researchers may choose to include measures where normative population values are available or where the HRQL instrument is available in their national language. Our review shows that the choice of an instrument is also country specific (e.g. in the Netherlands the EQ-5D is very often used and in the US the SF-36). Other factors such as user fees and instrument length will also be influential. Researchers may also choose instruments based on considerations that are specific to their study which may make generalizability difficult. For example, they may choose an injury specific instrument with greater responsiveness to change for their particular study question rather than a generic instrument.

The importance of using the same generic health instruments in multiple studies needs to be raised across the injury research community. In our view, the ideal measure to quantify the burden of injuries should include all dimensions relevant to the burden of injury, produce a 0-1 range, a utility or summary score, be responsive to changes over time and not be injury specific to enable comparisons with other diseases. We think that the HRQL instruments proposed by the EuroSafe group (EQ-5D and HUI) include the majority of the relevant dimensions for measurement of the burden of injury and the instruments are suitable for 'all injury' populations and all but the youngest age ranges $[45,46]$.

The Eurosafe recommendations were based on an assessment of whether all relevant health domains for injury patients are included, when measuring the functional consequences of injury. As a first criterion, all body functions, activities and participation domains of the International Classification of Functioning (ICF) were defined, that are relevant for a substantial proportion of injury patients: cognition, emotion, pain, problem solving, ambulation, use of hand/arm/fingers, self care, household activities, interpersonal interactions (including sexual activities), school and/or work, and recreation. Actually, none of the generic measures studied cover all the relevant domains. However, a combination of a measure focusing on the functional capacities of the patient on the one hand (such as the HUI) and a measure including social participation on the other hand (such as EQ-5D) provides the best compromise. To assess functional capacities, Functional Capacity Index (FCI [47]), the only available injury specific instrument could in principle be used, but validation studies of this measure have been few and inconclusive $[19,48]$. For this reason, the few studies 
Table 3 Reported utility scores and summarized study descriptions

\begin{tabular}{|c|c|c|c|c|c|}
\hline Study & Instrument & Sample size $(n)$ & time point & Index mean (SD) & Study population \\
\hline \multicolumn{6}{|l|}{ ALL INJURY PATIENTS } \\
\hline Keyes, US [19] & $\mathrm{QOL}$ & 854 & 24 months & 0.70 & Adults \\
\hline \multirow[t]{4}{*}{ Meerding, Netherlands [36] } & EQ-5D & 2904 & 2.5 months & 0.86 & all injury, age $15+$ \\
\hline & & & 2.5 months & 0.63 & hospitalized 15+ \\
\hline & & & 5 months & 0.74 & hospitalized $15+$ \\
\hline & & & 9 months & 0.74 & hospitalized 15+ \\
\hline \multirow[t]{3}{*}{ Polinder, Netherlands [3] } & EQ-5D & 525 & 2.5 months & 0.92 & all injury, age 4-15 \\
\hline & & 379 & 5 months & 0.96 & all injury, age 4-15 \\
\hline & & 366 & 9 months & 0.98 & all injury, age 4-15 \\
\hline \multirow[t]{4}{*}{ Polinder. Netherlands [37] } & EQ-5D & 3231 & 2.5 months & 0.60 & all injury, age $15+$ \\
\hline & & & 5 months & 0.70 & all injury, age $15+$ \\
\hline & & & 9 months & 0.76 & all injury, age $15+$ \\
\hline & & & 24 months & 0.73 & all injury, age $15+$ \\
\hline \multirow[t]{2}{*}{ Sutherland, UK [32] } & SF-6D & 200 & 2 months & 0.61 & Admitted, age 16-70 \\
\hline & & & 6 months & 0.62 & Admitted, age 16-70 \\
\hline \multirow[t]{2}{*}{ Watson, Australia [34] } & SF-6D & 186 & pre-injury & 0.91 & Admitted, age 18-74 \\
\hline & & 186 & 12 months & 0.73 & Admitted, age 18-74 \\
\hline \multicolumn{6}{|l|}{ SEVERE INJURY PATIENTS } \\
\hline \multirow[t]{2}{*}{ Gabbe, Australia [10] } & HAQ & 243 & discharge & 0.44 & ISS $>15$, age $15-80$ \\
\hline & & & 6 months & 0.54 & ISS $>15$, age $15-80$ \\
\hline \multirow[t]{4}{*}{ Holbrook US [12-14] } & QWB & 1048 & discharge & 0.40 & GCS $>11$, age $18+$ \\
\hline & & & 6 months & 0.63 & GCS $>11$, age $18+$ \\
\hline & & & 12 months & 0.67 & GCS $>11$, age $18+$ \\
\hline & & & 18 months & 0.68 & GCS $>11$, age $18+$ \\
\hline Holtslag, Netherlands [64] & EQ-5D & 335 & 15 months & 0.69 & ISS $>15$, age $15+$ \\
\hline Korosec, Slovenia [61] & EQ-5D & 98 & 24 months & 0.72 & ICU patients \\
\hline \multirow[t]{3}{*}{ Soberg, Norway [11] } & WHODAS $\| *$ & 105 & 6 weeks & 0.59 & ISS $>15$, age $18-67$ \\
\hline & & & 12 months & 0.72 & ISS > 15, age 18-67 \\
\hline & & & 24 months & 0.73 & ISS $>15$, age $18-67$ \\
\hline
\end{tabular}

* WHODAS II disability weights were reversed to utility scores.

$\mathrm{QOL}=$ Satisfaction with Quality of Life instrument; EQ-5 D = European Quality of Life instrument -5 dimensions; SF-6D = Medical Outcome Study Short Form-6 dimensions; HAQ = Health Assessment Questionnaire; QWB = Quality of Well Being Scale; WHODASII = World Health Organization Disability Assessment Schedule version II.

using the FCI conducted so far, were not included in our review.

Until improved evidence based recommendations become available, the EuroSafe guidance should be adopted across the injury field to facilitate comparisons between studies and to provide greater insight into functional outcome and recovery patterns after injury. Of course, depending on the type of injuries included in future studies researchers may continue to use different assessment periods and variability in follow-up. However, if researchers can adhere to the guidelines as closely as possible, the opportunity for the improved understanding of injury outcomes will be enhanced.

It is clear that given the current state of knowledge it is difficult to summarize the functional outcome of injuries amongst the general injury population, due to wide variety of study designs, instruments used, and timing of outcome assessments. Nevertheless, this review has provided an improved insight into functional outcomes and recovery patterns of injury patients. A high prevalence of health problems during and after the first year of injury was a common finding of the studies. Among 'all injury' groups recovery occurred predominately during the first year following injury, whereas some 'more severely' injured patients also recovered by a smaller amount during the second year. Two years post-injury both groups, on average, still showed large deficits from full recovery whether measured by population norms or differences from pre-injury health status.

Several authors have recently called for further comprehensive population-level research exploring outcomes after injury, particularly for the non selective 'all injury' group [6,7]. Our systematic review is one of the few studies that has considered the measurement of HRQL in 


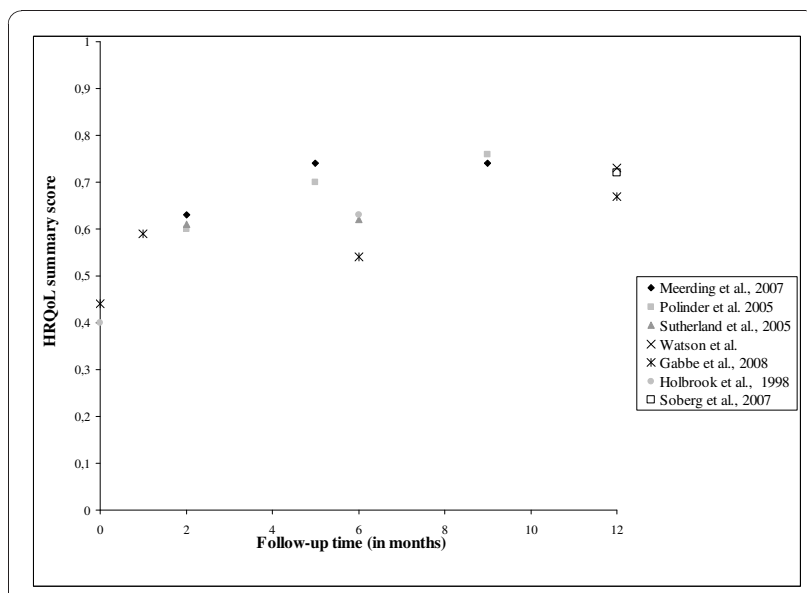

Figure 3 Reported HRQL summary scores and utility scores over time ( $\leq 12$ months) of general injury populations.

general injury populations. There is scarcity of well-standardized data on functional outcome after injury. In 2000, Krug et. al. concluded that very few populationbased data on non-fatal injury outcome were available [49]. In 2002, Garratt et all [50] concluded that there was relatively little development and evaluation of quality-oflife measures for trauma patients, compared to the almost exponential growth in other fields of health (care). Also, Segui-Gomez et al. reported on the limited application of quality-of-life measures in publications in the injury field [4]. The results of our review confirm these earlier conclusions. The earlier narrative literature search of the EuroSafe Group (2007) [1] identified only 17 studies. Four of the reviewed articles were not included in our review, because they were too injury specific (e.g. vertebral fractures [51]), or because they were cross-sectional in design and unsuited for measurement of outcomes [52-54]. Our review included 28 new studies which assessed injury-related recovery or disability. Furthermore, a systematic review of HRQL following major trauma among children reported similar findings with a large variety of $\mathrm{HRQL}$ measures used $(\mathrm{n}=14)$ [55].

With regards to future research in the injury field there is clearly a need for further empirical studies of injury outcomes which follow the general guidelines from the 2007 EuroSafe report [1]. Such studies should include a measure of pre-injury HRQL (retrospectively) using instruments that produces utility scores. Decisions regarding which HRQL measure to use is often influenced by a range of factors. Therefore, whilst researchers may use their own instruments, but should include the best validated generic instruments to ensure comparability of results across studies. The EQ5 D, combined with the HUI, is particularly recommended as an appropriate measure in 'all injury' studies due to its suitability, ease of use and also being free to use. When used, both utility scores and standard deviations should be reported. Longitudinal studies with multiple measurement points to study recovery patterns of injury patients should be a priority issue as many existing studies have had only one follow-up assessment. Since 'major trauma' patients often show further improvements after 12 months, studies focusing on such injuries should measure outcomes up to two years after injury.

Assessment of the impact of injury requires comparison with pre-injury HRQL or in the absence of such information age and gender specific population norms. Whilst population summary scores are often used new evidence suggests that such scores are significantly lower than the pre-injury summary scores of injury patients [27]. This implies that using population scores as a baseline results in an underestimation of the impact of the injury. Pre-injury scores were collected in the UK Burden of Injury Study and an Australian study though the validity of these 'pre'-injury data has been questioned as they are collected after the injury and may be prone to recall bias $[27,33,41]$. Comparison with population norms is also prone to bias as injured people are unlikely to be a random sample of the general population and adjustment may not be possible for unmeasured confounders.

\section{Conclusions}

In conclusion, this review shows that there is considerable variation in study design between studies measuring HRQL of general injury populations. It is also clear that recently developed guidelines are not yet being followed. Adherence to such guidelines would facilitate comparability across studies which would produce improved estimates of injury disability and recovery patterns. There is also a need for the development of bridging tables which would allow direct comparison of the results of studies using different instruments. Such tables would be a helpful step in supporting formal meta-analyses of the results of studies using different instruments.

There are still major gaps in our understanding of the impact of injury on personal and population health. Consistently collected empirical data across countries would support the production of more valid burden of injury calculations, cost-effectiveness analyses of injury prevention programs and trauma care, and support continuous quality improvement of care.

\section{Additional material}

Additional file 1: Search strategy PubMed

Additional file 2: Flow diagram of the reviewing process. 


\section{Acknowledgements}

Our work was done as part of the INTEGRIS project http://www.integris.nl, which is a European project enveloping an integrated injury registration system for Europe, funded by the European Union (EU). We thank the board of INTEGRIS for initially suggesting this study, and for their support and advice.

\section{Author details}

'Erasmus Medical Centre, Department of Public Health, Rotterdam, the Netherlands. ${ }^{2}$ College of Medicine, Swansea University, Swansea, UK. ${ }^{3}$ Faculty of Medicine, University of Oslo, Oslo, Norway.

\section{Authors' contributions}

SP had full access to all of the data in the study and takes responsibility for the integrity of the data and the accuracy of the data analysis. Review (literature search and data extraction): SP and EB. Study concept and design: $\mathrm{SP}, J \mathrm{H}, \mathrm{EFB}, \mathrm{VE}, \mathrm{JL}$ and RL. Analysis and interpretation of data: SP, JH, EFB, EB. Drafting of the manuscript: $\mathrm{SP}, \mathrm{EFB}, \mathrm{JH}$ and RL. Critical revision for important intellectual content and approval of the manuscript: SP, EFB, JH, RL, JL, VE. Study supervision: EFB.

\section{Competing interests}

The authors declare that they have no competing interests.

Received: 9 July 2010 Accepted: 23 December 2010 Published: 23 December 2010

\section{References}

1. Van Beeck EF, Larsen CF, Lyons RA, Meerding WJ, Mulder S, Essink-Bot ML: Guidelines for the conduction of follow-up studies measuring injuryrelated disability. J Trauma 2007, 62(2):534-550.

2. Haagsma JA, van Beeck EF, Polinder S, Hoeymans N, Mulder S, Bonsel GJ: Novel empirical disability weights to assess the burden of non-fatal injury. Inj Prev 2008, 14(1):5-10.

3. World Health Organization: International Classification of Functioning, Disabilities, and Health (ICF). Geneva: World Health Organization; 2001.

4. Segui-Gomez M, Mackenzie EJ: Measuring the Public Health impact of injuries. Epidemiol Rev 2003, 25:3-19.

5. Murray CJ, Lopez AD: Global mortality, disability, and the contribution of risk factors: Global Burden of Disease Study. The Lancet 1997, 349(9063): 1436-1442.

6. Derrett S, Black J, Herbison GP: Outcome after injury-a systematic literature search of studies using the EQ-5D. J Trauma 2009, 67(4):883-890.

7. Lyons RA: Measuring the burden of injury. Inj Prev 2008, 14(1):3-4.

8. Haagsma JA, Belt E, Polinder S, Lyons RA, Macey S, Atkinson M, Lund J, van Beeck EF: WP5 Injury disability indicators. Rotterdam: Department of Public Health, Erasmus Medical Center; 2009.

9. Lyons RA, Polinder S, Larsen CF, Mulder S, Meerding WJ, Toet H, Van Beeck E: Methodological issues in comparing injury incidence across countries. Int J Inj Contr Saf Promot 2006, 13(2):63-70.

10. World Health Organization Disability Assessment Schedule II. [http:// www.who.int/icidh/whodas/]

11. Aitken ME, Tilford JM, Barrett KW, Parker JG, Simpson P, Landgraf J, Robbins JM: Health status of children after admission for injury. Pediatrics 2002, 110(2 Pt 1):337-342.

12. Evanoff $B$, Abedin $S$, Grayson D, Dale AM, Wolf $L$, Bohr P: Is disability underreported following work injury? J Occup Rehabil 2002, 12(3):139-150.

13. Gillen M, Jewell SA, Faucett JA, Yelin E: Functional limitations and wellbeing in injured municipal workers: a longitudinal study. J Occup Rehabil 2004, 14(2):89-105.

14. Holbrook TL, Anderson JP, Sieber WJ, Browner D, Hoyt B: Outcome after major trauma: discharge and 6-month follow-up results from the Trauma Recovery Project. J Trauma 1998, 45(2):315-323.

15. Holbrook TL, Anderson JP, Sieber WJ, Browner D, Hoyt DB: Outcome after major trauma: 12-month and 18-month follow-up results from the Trauma Recovery Project. J Trauma 1999, 46(5):765-771.

16. Holbrook TL, Hoyt DB: The impact of major trauma: quality-of-life outcomes are worse in women than in men, independent of mechanism and injury severity. J Trauma 2004, 56(2):284-290.
17. Keyes KB, Wickizer TM, Franklin G: Two-year health and employment outcomes among injured workers enrolled in the Washington State Managed Care Pilot Project. Am J Ind Med 2001, 40(6):619-626.

18. Kiely JM, Brasel KJ, Weidner KL, Guse CE, Weigelt JA: Predicting quality of life six months after traumatic injury. J Trauma 2006, 61(4):791-798.

19. Barell V, Aharonson-Daniel L, Fingerhut LA, Mackenzie EJ, Ziv A, Boyko V, Abargel A, Avitzour M, Heruti R: An introduction to the Barell body region by nature of injury diagnosis matrix. Inj Prev 2002, 8(2):91-96.

20. Michaels AJ, Michaels CE, Smith JS, Moon CH, Peterson C, Long WB: Outcome from injury: general health, work status, and satisfaction 12 months after trauma. J Trauma 2000, 48(5):841-848; discussion 848-850.

21. Winthrop AL, Brasel KJ, Stahovic L, Paulson J, Schneeberger B, Kuhn EM: Quality of life and functional outcome after pediatric trauma. J Trauma 2005, 58(3):468-473, discussion 473-464.

22. Aitken ME, Jaffe KM, DiScala C, Rivara FP: Functional outcome in children with multiple trauma without significant head injury. Archives of physical medicine and rehabilitation 1999, 80(8):889-895.

23. Airey CM, Chell SM, Rigby AS, Tennant A, Connelly JB: The epidemiology of disability and occupation handicap resulting from major traumatic injury. Disability and rehabilitation 2001, 23(12):509-515.

24. Baldry Currens JA: Evaluation of disability and handicap following injury Injury 2000, 31(2):99-106.

25. Baldry Currens JA, Coats TJ: The timing of disability measurements following injury. Injury 2000, 31(2):93-98.

26. Braithwaite IJ, Boot DA, Patterson M, Robinson A: Disability after severe injury: five year follow up of a large cohort. Injury 1998, 29(1):55-59.

27. Lyons RA, Towner EE, Kendrick D, Christie N, Brophy S, Phillips CJ, Coupland C, Carter R, Groom L, Sleney J, et al: The UK burden of injury study - a protocol. [National Research Register number: M0044160889]. BMC Public Health 2007, 7:317.

28. Hetherington H, Earlam RJ, Kirk CJ: The disability status of injured patients measured by the functional independence measure (FIM) and their use of rehabilitation services. Injury 1995, 26(2):97-101.

29. Sutherland Aea: Recovery after musculoskeletal trauma in men and women. The Journal of Trauma 2005, 59(2):213-216.

30. Lamers LM, McDonnell J, Stalmeier PF, Krabbe PF, Busschbach JJ: The Dutch tariff: results and arguments for an effective design for national EQ-5 D valuation studies. Health Econ 2006, 15(10):1121-1132.

31. Gabbe BJ, Cameron PA, Williamson OD, Edwards ER, Graves SE, Richardson MD: The relationship between compensable status and longterm patient outcomes following orthopaedic trauma. Med J Aust 2007, 187(1):14-17.

32. Gabbe BJ, Simpson PM, Sutherland AM, Williamson OD, Judson R, Kossmann T, Cameron PA: Functional measures at discharge: are they useful predictors of longer term outcomes for trauma registries? Ann Surg 2008, 247(5):854-859.

33. Watson WL, Ozanne-Smith J, Richardson J: Retrospective baseline measurement of self-reported health status and health-related quality of life versus population norms in the evaluation of post-injury losses. Inj Prev 2007, 13(1):45-50.

34. Holtslag HR, Post MW, Lindeman E, Van der Werken C: Long-term functional health status of severely injured patients. Injury 2007, 38(3):280-289.

35. Meerding WJ, Looman CW, Essink-Bot ML, Toet H, Mulder S, van Beeck EF: Distribution and determinants of health and work status in a comprehensive population of injury patients. J Trauma 2004, 56(1):150-161.

36. Polinder S, Meerding WJ, van Baar ME, Toet H, Mulder S, van Beeck EF: Cost estimation of injury-related hospital admissions in 10 European countries. J Trauma 2005, 59(6):1283-1290, discussion 1290-1281.

37. Polinder S, van Beeck EF, Essink-Bot ML, Toet H, Looman CW, Mulder S, Meerding WJ: Functional outcome at 2.5, 5, 9, and 24 months after injury in the Netherlands. J Trauma 2007, 62(1):133-141.

38. Janssens L, Gorter WJ, Ketelaar M, Kramer WLM, Holtslag HR: Long-term health condition in major pediatric trauma: a pilot study. J Pediatric Surgery 2009, 44:1591-1600.

39. Macpherson AK, Rothman L, McKeag AM, Howard A: Mechanism of injury affects 6-month functional outcome in children hospitalized because of severe injuries. J Trauma 2003, 55(3):454-458. 
40. Aitken ME, Tilford JM, Barrett KW, Parker JG, Simpson P, Landgraf J, Robbins JM: Health status of children after admission for injury. Pediatrics 2002, 110:337-342.

41. Gabbe BJ, Cameron PA, Graves SE, Williamson OD, Edwards ER: Preinjury status: are orthopaedic trauma patients different than the general population? J Orthop Trauma 2007, 21(4):223-228.

42. Polinder $\mathrm{S}$, van Beeck EF, Essink-Bot ML, Toet $\mathrm{H}$, Looman CW, Mulder $\mathrm{S}$, Meerding WJ: Functional outcome at 2.5, 5, 9, and 24 months after injury in the Netherlands. J Trauma 2007, 62:133-141.

43. Soberg HL, Bautz-Holter E, Roise O, Finset A: Long-term multidimensional functional consequences of severe multiple injuries two years after trauma: a prospective longitudinal cohort study. J Trauma 2007, 62(2):461-470.

44. Mokkink LB, Terwee CB, Knol DL, Stratford PW, Alonso J, Patrick DL, Bouter LM, de Vet HCW: The COSMIN checklist for evaluating the methodological quality of studies on measurement properties: $\mathrm{A}$ clarification of its content. BMC Medical Research Methodology 2010, 10(8)

45. Haagsma JA, Polinder S, Havelaar AH: Analysis and documentation of the results of the reveiw of existing burden of disease methods. Rotterdam: Department of Public Health, Erasmus Medical Center; 2010.

46. Polinder S, Haagsma JA, Bonsel G, Essink-Bot ML, Toet H, van Beeck EF: The measurement of long-term health-related quality of life after injury: comparison of EQ-5 D and the health utilities index. Inj Prev 16(3):147-153.

47. Mackenzie EJ, Damiano A, Miller T, Luchter S: The development of the Functional Capacity Index. J Trauma 1996, 41(5):799-807.

48. Schluter PJ, Neale R, Scott D, Luchter S, McClure RJ: Validating the functional capacity index: a comparison of predicted versus observed total body scores. J Trauma 2005, 58(2):259-263.

49. Krug EG, Sharma GK, Lozano R: The global burden of injuries. Am J Public Health 2000, 90(4):523-526.

50. Garratt A, Schmidt L, Mackintosh A, Fitzpatrick R: Quality of life measurement: bibliographic study of patient assessed health outcome measures. BMJ 2002, 324:1417-1421.

51. Badia X, Diez-Perez A, Alvarez-Sanz C, Diaz-Lopez B, Diaz-Curiel M, Guillen F, Gonzalez-Macias J, Spanish GSG: Measuring quality of life in women with vertebral fractures due to osteoporosis: a comparison of the OQLQ and QUALEFFO. Qual Life Res 2001, 10(4):307-317.

52. Inaba K, Goecke M, Sharkey P, Brenneman F: Long-term outcomes after injury in the elderly. J Trauma 2003, 54(3):486-491.

53. Vles WJ, Steyerberg EW, Essink-Bot ML, van Beeck EF, Meeuwis JD, Leenen LP: Prevalence and determinants of disabilities and return to work after major trauma. J Trauma 2005, 58(1):126-135.

54. Zelle B, Stalp M, Weihs C, Müller F, Reiter F, Krettek C, Pape H: Validation of the Hannover Score for Polytrauma Outcome (HASPOC) in a sample of 170 polytrauma patients and a comparison with the 12-Item Short-Form Health Survey [in German]. Chirurg 2003, 74:361-369.

55. Janssens L, Gorter JW, Ketelaar M, Kramer WL, Holtslag HR: Health-related quality-of-life measures for long-term follow-up in children after major trauma. Qual Life Res 2008, 17(5):701-713.

56. Vazquez Mata G, Rivera Fernandez R, Perez Aragon A, Gonzalez Carmona A, Fernandez Mondejar E, Navarrete Navarro P: Analysis of quality of life in polytraumatized patients two years after discharge from an intensive care unit. J Trauma 1996, 41(2):326-332.

57. Gofin R, Adler B: A seven item scale for the assessment of disabilities after child and adolescent injuries. Inj Prev 1997, 3(2):120-123.

58. Gofin R, Hass T, Adler B: The development of disability scales for childhood and adolescent injuries. J Clin Epidemiol 1995, 48(7):977-984

59. Brenneman FD, Redelmeier DA, Boulanger BR, McLellan BA, Culhane JP: Long-term outcomes in blunt trauma: who goes back to work? J Trauma 1997, 42(5):778-781.

60. Kopjar B: The SF-36 health survey: a valid measure of changes in health status after injury. Inj Prev 1996, 2(2):135-139.

61. Korosec Jagodic H, Jagodic K, Podbregar M: Long-term outcome and quality of life of patients treated in surgical intensive care: a comparison between sepsis and trauma. Crit Care 2006, 10(5):R134.

62. Alves ALA, Salim FM, Martinez EZ, Passos ADC, de Carlo MMRP, Scarpelini S: Quality of life in trauma victims six months after hospital discharge. Rev Saude Publica 2009, 43:1-6.
63. Pirente N, Gregor A, Bouillon B, Neugebauer E: Quality of life of severely injured patients 1 year after trauma. A matched-pair study compared with a healthy control group [in German]. Unfallchirurg 2001, 104:57-63.

64. Holtslag HR, van Beeck EF, Lindeman E, Leenen LP: Determinants of longterm functional consequences after major trauma. J Trauma 2007, 62:919-927.

65. Dimopoulou I, Anthi A, Mastora Z, Theodorakopoulou M, Konstandinidis A Evangelou E, Mandragos K, Roussos C: Health-related quality of life and disability in survivors of multiple trauma one year after intensive care unit discharge. Am J Phys Med Rehabil 2004, 83(3):171-176.

66. Stalp M, Koch C, Regel G, Krettek C, Pape HC: Development of a standardized instrument for quantitative and reproducible rehabilitation data assessment after polytrauma (HASPOC) (in German). Chirurg 2001, 72:312-318.

67. Stalp M, Koch C, Ruchholtz S, Regel G, Panzica M, Krettek C, Pape HC: Standardized outcome evaluation after blunt multiple injuries by scoring systems: a clinical follow-up investigation 2 years after injury. J Trauma 2002, 52(6):1160-1168.

\section{Pre-publication history}

The pre-publication history for this paper can be accessed here: http://www.biomedcentral.com/1471-2458/10/783/prepub

doi:10.1186/1471-2458-10-783

Cite this article as: Polinder et al:: A systematic review of studies measuring health-related quality of life of general injury populations. BMC Public Health 2010 10:783.

\section{Submit your next manuscript to BioMed Central and take full advantage of:}

- Convenient online submission

- Thorough peer review

- No space constraints or color figure charges

- Immediate publication on acceptance

- Inclusion in PubMed, CAS, Scopus and Google Scholar

- Research which is freely available for redistribution

Submit your manuscript at www.biomedcentral.com/submit
C) Biomed Central 